\title{
Current concepts in the management of necrotizing fasciitis
}

\author{
Evangelos P. Misiakos*, George Bagias, Paul Patapis, Dimitrios Sotiropoulos, Prodromos Kanavidis and \\ Anastasios Machairas
}

3rd Department of Surgery, Attikon University Hospital, University of Athens School of Medicine, Athens, Greece

Edited by:

Hubert Scheuerlein,

Universitätsklinikum Jena, Germany

Reviewed by:

Carolina Isabella Alexandra

Pape-Köhler, University of

Witten/Herdecke, Germany

Markus Philipp Hussein Ghadimi,

Universitätsklinik Düsseldorf,

Germany

\section{*Correspondence:}

Evangelos P. Misiakos, 3rd

Department of Surgery, Attikon

University Hospital, University of

Athens School of Medicine, 76 Aigeou

Pelagous Street, Agia Paraskevi,

Athens 15 341, Greece

e-mail:misiakos@med.uoa.gr,

emisiakos@yahoo.com
Necrotizing fasciitis (NF) is a severe, rare, potentially lethal soft tissue infection that develops in the scrotum and perineum, the abdominal wall, or the extremities. The infection progresses rapidly, and septic shock may ensue; hence, the mortality rate is high (median mortality $32.2 \%$ ). Prognosis becomes poorer in the presence of co-morbidities, such as diabetes mellitus, immunosuppression, chronic alcohol disease, chronic renal failure, and liver cirrhosis. NF is classified into four types, depending on microbiological findings. Most cases are polymicrobial, classed as type I. The clinical status of the patient varies from erythema, swelling, and tenderness in the early stage to skin ischemia with blisters and bullae in the advanced stage of infection. In its fulminant form, the patient is critically ill with signs and symptoms of severe septic shock and multiple organ dysfunction. The clinical condition is the most important clue for diagnosis. However, in equivocal cases, the diagnosis and severity of the infection can be secured with laboratory-based scoring systems, such as the laboratory risk indicator for necrotizing fasciitis score or Fournier's gangrene severity index score, especially in regard to Fournier's gangrene. Computed tomography or ultrasonography can be helpful, but definitive diagnosis is attained by exploratory surgery at the infected sites. Management of the infection begins with broad-spectrum antibiotics, but early and aggressive drainage and meticulous debridement constitute the mainstay of treatment. Postoperative management of the surgical wound is also important for the patient's survival, along with proper nutrition. The vacuum-assisted closure system has proved to be helpful in wound management, with its combined benefits of continuous cleansing of the wound and the formation of granulation tissue.

Keywords: necrotizing fasciitis, Fournier's gangrene, gas gangrene, surgical debridement

\section{INTRODUCTION}

The term necrotizing fasciitis (NF) describes a group of relatively uncommon, but life-threatening infections of the skin, soft tissues, and muscles, which tend to progress rapidly through the fascia planes, causing gradual destruction of the fascia at a rate reaching $2-3 \mathrm{~cm} / \mathrm{h}$. Developing in the lower or upper extremities, the perineum and genital area (Fournier's gangrene) and in the abdominal wall, its swift clinical course is correlated with polymicrobial infection and synergy, which usually co-exists $(1,2)$. The majority of cases present anaerobic bacteria that proliferate in a hypoxic environment and produce gas, which accumulates in the soft tissue spaces, giving the characteristic image of gas gangrene on plain X-rays and computed tomography (CT) scans (3).

Early diagnosis of NF is mandatory. Any delay could prove fatal, given its association with more extensive surgery, higher rates of amputation, and higher mortality rates. Furthermore, if left untreated, the infection could lead to systemic inflammatory response syndrome (SIRS).

Necrotizing fasciitis was first recognized in 500 BC, when Hippocrates reported a clinical description of a complication of erysipelas disease, resembling the current description of NF (4). In France, Claude Colles, chief surgeon of the Hotel Dieu in Lyon, described a condition in 1783 that was very similar to modern descriptions of NF (5). The first description of "modern" NF was made by Joseph Jones, a military surgeon of the army of the Confederate States of America. In 1871, he reported 2,642 cases of gas gangrene treated in hospital during the American Civil War, with a mortality rate of approximately $46 \%$ (6). In 1883, Jean Alfred Fournier described a syndrome with necrosis of the perineum in five men; this type of NF was subsequently given his name and is known as Fournier's gangrene (7). In 1924, Meleney reported an association with beta-hemolytic streptococcus A in a study of a series of hospitalized cases in Beijing. Thereafter, these cases were described for several decades as Meleney's gangrene (8). In 1952, the term "necrotizing fasciitis" was proposed by Wilson, as a more accurate description of this disease (9). The late 1980s witnessed a renewed interest in this pathology. Stevens reported that, among 20 patients who presented with streptococcal shock, 11 were diagnosed as having NF. The disease was popularized by the media as "flesh-eating bacteria syndrome" (10).

\section{EPIDEMIOLOGY}

The annual incidence of NF is estimated at 500-1,000 cases annually, and its prevalence globally has been reported to be 0.40 cases 
per 100,000 population (11). It is seen to have a predilection for men, with a male-to-female ratio of $3: 1$; this ratio is mainly correlated with the increased incidence of Fournier's gangrene in men. The disease affects all age groups, although middle-aged and elderly patients (over 50 years of age) are more likely to be infected (1). The median mortality ratio of NF is a controversial issue. In their review of the literature, Goh et al. concluded that the median mortality ratio was $21.5 \%$ (12). However, its range in the literature is extensive, varying from 8.7 to $76 \%$ (13). In regard to NF of the extremities, the mortality rate is slightly lower than that recorded for abdominal and perineal infections $(14,15)$. Patients with Fournier's gangrene that has not spread to the abdominal wall tend to have a better survival. As a general rule, without treatment, the mortality rate approaches $100 \%$. Anaya et al. (16) have demonstrated that infection of the lower extremities is the most common site of NF $(57,8 \%)$, followed by the abdomen and the perineum. NF of the upper limbs is rare compared to that of the lower limbs (17).

\section{ETIOLOGY}

Trauma is the most common identifiable etiology. The majority of patients have a history of minor or major traumas, generally involving external injuries and surgical wounds. Appendicitis with perforation, infection following the repair of an incarcerated hernia, perforated diverticulitis, necrotic cholecystitis, gastroduodenal perforation, small bowel perforation, and obstructive colon cancer with perforation rank among the most frequent causes of complicated intra-abdominal infections that can lead to NF. Notably, the incidence of NF resulting from a surgical wound in the chest wall is greater than that recorded from analogous wounds in the lower abdominal wall. Such cases present a high risk of osteomyelitis, which substantially increases the mortality of these patients.

Fournier's gangrene is often the result of surgical wounds, skin abscess drainage, and pressure sores. It can also present as a complication of colorectal disease due to anorectal infection, ischiorectal abscesses, and colon perforations. Other causes include a possible urethral stricture and a trauma from an indwelling Foley catheter. In women, it has commonly been ascribed to Bartholin abscesses or vulval skin infections.

In Asia, consumption of raw or undercooked seafood or injury by fish fins can lead to NF (12). In this group of infections, bacteria such as Vibrio spp., Aeromonas spp., and Shewanella spp. are commonly involved and are usually known as "marine bacteria" (18).

\section{CO-MORBIDITIES AND RISK FACTORS}

The most frequent co-morbidity in patients with NF is diabetes mellitus. The prevalence of diabetes mellitus in patients with any type of NF ranges between 40 and $60 \%(12,19)$. Other common co-morbidities include liver cirrhosis, chronic heart failure, obesity, alcohol abuse, immunodeficiency, systemic lupus erythematosus, Addison's disease, pre-existing hypertension, and peripheral vascular disease (20,21). A septic condition and hypotension at the time of admission are significant predicting factors for mortality and outcome. Chronic renal failure is another indisputable predisposing factor for higher mortality in patients with NF. Elevated serum creatinine, along with elevated blood urea, is also strongly associated with higher mortality rates (22).

The use of non-steroidal anti-inflammatory drugs (NSAIDs) or steroid drugs can suppress fever, thereby hampering the diagnosis of NF (8). Furthermore, Martinschek et al. have demonstrated that an increase of serum creatine kinase and lactate parameters, as well as a decrease of serum antithrombin III, proved by a low INR, are significant parameters for an unfavorable outcome, particularly in regard to Fournier's gangrene (20). Other risk factors, such as systemic acidosis, low hematocrit, and albumin levels, are also strongly linked with a high mortality, while concomitant conditions increase the mortality rate further (23). Patients displaying accompanying diseases are usually characterized as critically ill and require prolonged intensive care. Diabetes, in particular, is a disease, which often combines many of the above co-morbidities, and is hence susceptible to the development of NF (24). However, the presence of diabetes mellitus has not been proven to affect mortality (25).

Advanced age constitutes another risk factor for higher incidence and mortality, although somewhat controversial. Large population-based studies have shown that advanced age is a strong, independent predictor of mortality (26). A study by Rea and Wyrick reported a mortality rate of $67 \%$ in patients over 50 years of age and $4 \%$ in patients under that age (27). Other studies have concurred that advanced age is a risk factor for higher mortality, but only when accompanied by other risk factors such as renal failure, or delayed surgical debridement (24). This combination is also associated with advanced disease and a more fulminant infection.

Whether or not patients with NF show a gender predilection with regard to mortality is also a topic of debate. Fournier's gangrene shows male predominance with a reported rate of $96 \%$ (28). Czymek et al. found that mortality was significantly higher among females (50\% F vs. 7.7\% M) (29). However, studies involving a larger study population have concluded that there is no statistical correlation between female gender and increased mortality (30).

The extension and variability of infection are assumed to increase mortality. NF can affect an entire extremity within $24 \mathrm{~h}$ (31), but it can also show slow progression over a period of several weeks. In some patients, the disease remains dormant and unexpectedly spreads rapidly without any readily apparent reason (24). The factors that lead to the fulminant form of NF, with a potentially lethal outcome within $24 \mathrm{~h}$, remain unidentified (32). Some studies indicate that the spread of gangrene does not relate to a poorer prognosis. Notwithstanding, the extension of gangrene to the abdominal wall has been reported to be directly related to increased mortality (33).

\section{PATHOPHYSIOLOGY}

Infection begins in the hypodermis or the superficial fascia, as the more superficial layers (dermis and epidermis) are not affected at the beginning (34). The synergistic action of the virulence factors of bacteria and the specific factors of the host are implicated in the development of NF. The extension of the infection and necrosis is facilitated by the synergy between the different bacteria and toxins and the enzymes they produce (35). An anaerobic environment promotes growth of anaerobic bacteria. Necrosis of 
the hypodermis and superficial fascia is directly related to bacterial enzymes that destroy the fascia and fat, and secondarily to vascular origin. Invasive bacteria cause thrombosis of the nutrient vessels, which are located in the hypodermis, leading to tissue ischemia aggravated by the presence of edema. Tissue ischemia promotes infectious dissemination leading to skin necrosis at a later stage. It also explains the intense pain phenomena that are usually observed, especially when the nerve branches are also affected. Such cases also display signs of regional hypoesthesia/anesthesia. The fascial and hypodermic necrotic spread is greater than the overlying skin changes. Lymphangitis and lymphadenopathy are rare due to thrombosis of the vessels. Gas formed by anaerobic bacteria may lead to crepitus.

\section{MICROBIOLOGY}

Recent studies have concluded that NF can be classified into four types, according to microbiological findings (35)(Table 1). The most common is type I, also known as the polymicrobial type. Accounting for 70-90\% of cases, it typically affects patients with several co-morbidities, such as diabetes mellitus. Two or more pathogens are implicated in this infection (with an average of 4.4 species) and it is mostly found in the trunk and perineum.

Type II, otherwise known as monomicrobial, is defined as infection with beta-hemolytic Streptococcus A (Streptococcus pyogenes). S. pyogenes is commonly found in young and healthy patients with NF of the extremities. Its pathogenesis is explained by the several virulence factors produced by this organism (36). In some cases, the infection can be associated with Staphylococcus aureus. S. aureus secretes toxins, which cause leukocyte destruction and tissue necrosis. Found in the fulminant forms of NF, S. aureus is not easy to manage, especially when the responsible pathogen is the methicillin-resistant $S$. aureus (MRSA), which is the case in $10-30 \%$ of all patients. Typically, these infections occur after small incisions, and appear to be highly correlated with the use of NSAIDs (37). Specifically observed in patients without serious co-morbidities, the infection is most often found in the limbs. The risk of toxic shock syndrome is increased in such cases, and the outcome is unfavorable.

Type III includes monomicrobial infections involving the Clostridium species or Gram-negative bacteria. Clostridium species are anaerobic bacteria that can be produced by external injuries (deep wound or crush injury causing local devascularization) or surgical wounds (intestinal and obstetric). Clostridium infections are currently more frequent among drug addicts (38), and $C$. perfringens is the most common bacterium of the $C$. species. Vibrios spp. infections can also lead to type III NF. Vibrio vulnificus is a marine bacterium frequently isolated in Asia (21). Aeromonas hydrophila is found in freshwater or low salinity water and in the soil. The clinical symptoms of infections by these two bacteria are similar; hemorrhagic blisters, lesions, and purpuric necrosis are the dominant symptoms, along with the extremely rapid spread of disease.

Finally, type IV is the result of fungal infections, mainly Candida spp. and Zygomycetes. This type is found mainly in the immunocompromised host. Infections by these fungi often occur after trauma; the clinical image is aggressive and rapidly extensive, particularly in immunocompromised patients.

Microbiological diagnosis is obtained in almost $75 \%$ of all cases of NF (39), and is based on the good quality of the pre- and intraoperative samples and blood cultures. Blood cultures are positive in $25 \%$ of all cases, while cultures obtained from the site of injury during surgical debridement result as positive in $80 \%$ of cases $(40)$.

\section{DIAGNOSIS}

\section{CLINICAL SIGNS AND SYMPTOMS}

Patients with NF usually present with the classic triad of symptoms: local pain, swelling, and erythema (12). Tachycardia (>100 beats/min) and fever are the most common vital sign abnormalities, followed by hypotension ( $\mathrm{SAP}<100 \mathrm{mmHg}$ ) and tachypnea (>20/min). These vital sign abnormalities, along with the skin erythema, are most useful in securing the diagnosis of NF from other soft tissue infections (41). The infected site displays tenderness, sclerosis, skin necrosis, and hemorrhagic bullae (42).

Depending on the development of the infection, the clinical image described above may not always be evident. Consequently, two groups of symptoms are considered, namely early and advanced symptoms (43). The most common early signs are erythema, local warmth, skin sclerosis, and edema. However, in the fulminant form of disease, the patient is critically ill with signs and symptoms of severe septic shock and multiple organ dysfunction syndrome, along with extensive necrosis of soft tissue. In this case, the clinical picture deteriorates rapidly within a few hours; pain is severe and usually manifests before the cutaneous signs. Remarkably, pain seems to be disproportionate to the clinical findings.

In contrast, the subacute form of the disease has a relatively slow clinical course, which may endure for days or weeks. The early clinical status of the subacute form is the result of an existing

Table 1 | Classification of responsible pathogens according to type of infection.

\begin{tabular}{|c|c|c|c|}
\hline Microbiological type & Pathogens & Site of infection & Co-morbidities \\
\hline Type I (polymicrobial) & Obligate and facultative anaerobes & Trunk and perineum & Diabetes mellitus \\
\hline Type II (monomicrobial) & Beta-hemolytic streptococcus A & Limbs & \\
\hline \multirow[t]{4}{*}{ Type III } & Clostridium species & Limbs, trunk, and perineum & Trauma \\
\hline & Gram-negative bacteria & & Seafood consumption (for Aeromonas) \\
\hline & Vibrios spp. & & \\
\hline & Aeromonas hydrophila & & \\
\hline \multirow[t]{2}{*}{ Type IV } & Candida spp. & Limbs, trunk, perineum & Immunosuppression \\
\hline & Zygomycetes & & \\
\hline
\end{tabular}


condition leading to infection. The patient often presents with a skin infection, such as folliculitis or abscess, gangrene on the extremities, pressure sore(s), or a complicated surgical wound. Erythema or skin sclerosis is present at the site of infection. The patient usually feels pain at the site of the injury, and this is a very strong diagnostic hint. However, local nerves can also be infected, usually resulting in the partial loss of sensation (44).

As the infection develops, the pain becomes more intense. The clinical image is characterized by symptoms of general toxicity including fever, dehydration, confusion, dizziness, diarrhea, nausea, vomiting, weakness, and malaise (19). If the patient remains undiagnosed or untreated, the clinical status deteriorates rapidly. The cutaneous symptoms may progress to blisters and bullae, ultimately leading to circumscribed necrosis of the skin. Initially, the bullae contain serous fluid, but, as the infection progresses, they may become hemorrhagic. Gas formation can lead to crepitus in the overlying skin, indicating anaerobic infection, such as C. perfringens. This classical skin condition does not normally present until day five or later (45).

Symptoms of septic shock or MODS frequently appear in the late phase of its subacute form. As a result, the patient displays hypotension, elevated white blood cell count, metabolic acidosis, coagulopathy, changes in mental status, and weakness. In this late stage of the disease, the patient looks apathetic and indifferent. Additional symptoms pertaining to co-morbidities may also coexist.

The symptoms of disease are not characteristic; hence, it is often misdiagnosed as cellulitis or abscess. The most consistent feature of early NF is pain, which is not in proportion to the swelling or erythema (46). Moreover, as a consequence of the enzymatic and toxin action, tenderness to palpation extends beyond the area of apparent involvement, to spread along fascial plains. In addition, the margins of involvement are usually indistinct, and lymphangitis is rarely present, given that the infection is in the deep fascia rather than the skin (47).

Cases with upper limb infection do not always present a typical picture. In this instance, patients may appear systemically well (21). Another cause that complicates diagnosis is the absence of fever in most cases. Several drugs, such as NSAIDs, steroids, and antibiotics can lower body temperature and mask fever. For that reason, the absence of pyrexia does not necessarily exclude NF $(12,41)$.

Fournier's gangrene has a slightly different clinical course. It usually begins with pain and itching of the perineum and scrotal skin. In genitourologic types of Fournier's gangrene, the pathogens pass through the Buck's fascia of the penis and spread along the Darto's fascia of the scrotum and penis, Colle's fascia of the perineum, and Scarpa's fascia of the lower abdominal wall (19). Additional necrosis of the superficial fascia and fat produces a thin watery malodorous fluid and crepitus. Similarly, patients may present high fever, anxiety, altered mental status, leukocytosis, shock, and tachypnea, when shock is about to develop. Once clinical signs become obvious, the appearance resembles the late phase of NF, with visible bruising, bullae and cutaneous necrosis due to the extension of the necrotizing process.

\section{BEDSIDE AND LABORATORY TESTS}

Laboratory results in this disease are not usually specific. However, certain laboratory findings can help the clinician to differentiate NF from other skin diseases, such as necrotizing soft tissue infection. Specifically, leukocytosis is a common feature in patients with NF (48), and white blood cell count in excess of $20,000 / \mathrm{L}$ is highly suspect. Blood urea nitrogen $>18 \mathrm{mg} / \mathrm{dL}$ and serum creatinine $>1.2 \mathrm{mg} / \mathrm{dL}$ reflect ongoing renal failure, which is typically present in these patients. Serum creatine kinase is also elevated (CK) in patients with severe sepsis and MODS (48). Majeski et al. suggested that C-reactive protein $>16 \mathrm{mg} / \mathrm{dL}$ or creatine kinase $>600$ IU/L generally precludes group A $\beta$-hemolytic streptococcal infection (49). However, this recommendation is rarely followed by clinicians.

Several laboratory-based scoring systems have been proposed for establishing early diagnosis of NF (50). The Laboratory Risk Indicator for Necrotizing Fasciitis (LRINEC) proposed by Wong et al. (51-53) is one such example (Table 2). In addition to enabling early recognition of the disease, this score can also facilitate the classification of patients into risk categories, and help in the allocation of diagnostic resources. Another scoring system is the Fournier's Gangrene Severity Index (FGSI) that has shown remarkable success in helping to determine whether or not the patient requires surgical debridement (54).

Bedside tests, imaging tests [CT or magnetic resonance imaging (MRI)], or frozen section biopsy can be carried out in patients with equivocal clinical findings and a moderate or high risk of NF based on the LRINEC score ( $>5$ ). The finger test and frozen section biopsy are used as complementary diagnostic modalities in patients with an equivocal diagnosis. Surgical exploration is regarded as the mainstay for investigation and treatment. The finger test is a bedside procedure performed under local anesthesia by which, a $2-\mathrm{cm}$ incision is made down to the deep fascia, at which level gentle probing of the index finger is applied. The presence

Table 2 | LRINEC scoring system for necrotizing fasciitis.

\begin{tabular}{lr}
\hline Variable & Score \\
\hline $\operatorname{CRP}(\mathrm{mg} / \mathrm{L})$ & \\
$\quad>150$ & 4 \\
$\mathrm{WBC}(\mathrm{g} / \mathrm{L})$ & \\
$\quad<15$ & 0 \\
$15-25$ & 1 \\
$>25$ & 2 \\
Hemoglobin $(\mathrm{g} / \mathrm{dL})$ & \\
$\quad>13.5$ & 0 \\
$11-13.5$ & 1 \\
$\quad<11$ & 2 \\
Sodium $(\mathrm{mmol} / \mathrm{L})$ & \\
$\quad<135$ & 2 \\
Creatinine ( $\mu \mathrm{mol} / \mathrm{L})$ & \\
$\quad>141$ & 2 \\
Glucose $(\mathrm{mmol} / \mathrm{L})$ & 1 \\
$\quad<10$ &
\end{tabular}


of characteristic "dishwater pus," along with the lack of bleeding and lack of tissue resistance to blunt finger dissection are positive findings correlated with NF. Another useful bedside test is an incisional biopsy down to the fascial level with an immediate frozen section, culture, and Gram stain $(55,56)$.

The combination of surgical exploration and microbiological and histopathological analysis of $1 \mathrm{~cm}^{3}$ of soft tissue is considered the gold standard for confirming diagnosis, when the latter is ambivalent.

\section{IMAGING TESTS}

Imaging investigation can help to establish the diagnosis of NF especially in equivocal cases. Although plain radiography has low sensitivity and specificity, it is capable of showing gas formation in the soft tissue (33), which is present in almost half of all patients, and it strongly indicates infection by the Clostridium species. CT and MRI are more sensitive and specific than plain radiography. A CT scan can show the extent of tissue infection, fascial swelling, inflammation, and gas formation. An MRI scan provides better accuracy than CT, though not widely used, due to cost. Ultrasonography is also a feasible option, providing useful information concerning the nature and extent of infection, especially when the diagnosis is unclear (57). In terms of diagnosis, the most significant finding is the hyperechoic foci with reverberation artifact and dirty shadowing at the site of infection (57), representing the subcutaneous gas. However, ultrasonography requires a highly skilled operator; this requirement hampers its use in the ICU, where patients with NF are usually treated.

\section{TREATMENT}

\section{ANTIBIOTIC TREATMENT}

Since ischemia and hypoxia compromise the adequate delivery of antibiotics to the infection site, conservative treatment with antibiotics alone has little value in the management of NF (58). However, they play a significant role in surgical management of the infection. Patients should be immediately treated with broad-spectrum antibiotics, when NF is suspected. The empirical usage of antibiotics is based on the microbiological classification of NF. Antibiotic treatment of a polymicrobial infection should be based on history, Gram stain, and culture. Initial treatment includes ampicillin or ampicillin-sulbactam combined with metronidazole or clindamycin (59). Anaerobic coverage is quite important for type 1 infection; metronidazole, clindamycin, or carbapenems (imipenem) are effective antimicrobials. Broad gram-negative coverage is necessary as an initial empirical therapy for patients who have recently been treated with antibiotics, or been hospitalized. In such cases, antibiotics such as ampicillinsulbactam, piperacillin-tazobactam, ticarcillin-clavulanate acid, third or fourth generation cephalosporins, or carbapenems are used, and at a higher dosage.

Type 2 disease is treated with antibiotics against $S$. pyogenes and $S$. aureus, which usually coexist with the former. Hence, first or second generation of cephalosporins are used for the coverage of methicillin-sensitive Staphylococcus aureus (MSSA). MRSA tends to be covered by vancomycin, or daptomycin and linezolid in cases where $S$. aureus is resistant to vancomycin. Some studies suggest that clindamycin is superior to penicillin in managing streptococcal infections (60), but this has yet to be satisfactorily proven. Another study has proposed that clinicians should consider adding clindamycin to the beta-lactam antibiotic regimen when NF or myositis is present (61).

Type 3 NF should be managed with clindamycin and penicillin, which cover the Clostridium species. If Vibrio infection is suspected, the early use of tetracyclines (including doxycycline and minocycline) and third-generation cephalosporins is crucial for the survival of the patient, since these antibiotics have been shown to reduce the mortality rate drastically (59).

Finally, type 4 NF can be treated with amphotericin B or fluoroconazoles, but the results of this treatment are generally disappointing.

As in every empirical antibiotic therapy, the dosage should be tapered, based on the results of the initial blood, wound, and tissue cultures, but continued until the infection is under control and for at least $48 \mathrm{~h}$ after clinical and hemodynamic stabilization of the patient has been achieved. Antibiotics should be administered for up to 5 days after local signs and symptoms have resolved (62). The mean duration of antibiotic therapy for NF is 4-6 weeks.

Intravenous immunoglobulin (IVIG) has recently been described as a reasonable and desirable option for neutralizing streptococcal toxins (63). There is evidence that a high dose of IVIG may prove beneficial in severe streptococcal infections (64), but this has yet to be demonstrated with randomized studies.

\section{SURGICAL MANAGEMENT}

Emergency surgical debridement of the affected tissues is the primary management modality for NF. Surgical debridement, necrosectomy, and fasciotomy are the main aspects of surgical treatment. Surgical intervention is life-saving and must be performed as early as possible, since a delay in treatment beyond $12 \mathrm{~h}$ in fulminant forms of NF can prove fatal. Surgical debridement should be repeated during the next $24 \mathrm{~h}$ or later, depending on the clinical course of the necrotizing infection and vital functions. Many studies have pointed out that timing and the extent of the first debridement are the most important risk factors in terms of increased mortality rate. Mock et al. have shown that the relative risk of death was 7.5 times greater in cases of restricted primary debridement (65), whereas, other studies reported that the mortality rate was nine times greater when primary surgery was performed $24 \mathrm{~h}$ after the onset of symptoms (19).

Surgical management is indicated especially for patients displaying intense pain and skin color change, such as edema and/or ecchymoses, or signs of skin ischemia with blisters and bullae (Figure 1). Patients must be operated on urgently when they present with altered mental status, hypotension, an elevated band formed in the differential WBC count, and metabolic acidosis. These clinical and laboratory signs indicate that the patient has developed SIRS or MODS, and the NF score has risen to phase 3. The mortality rate in this phase is extremely high, reaching almost $70 \%(24)$.

Incisions are performed parallel to Langer's lines to achieve better surgical wound healing and less scarring. Surgery also minimizes the overall tissue loss as it inhibits infection spread to the fascial plane, reducing the need for amputation (66). After the release of pus and/or hemorrhagic fluid through incisions, 


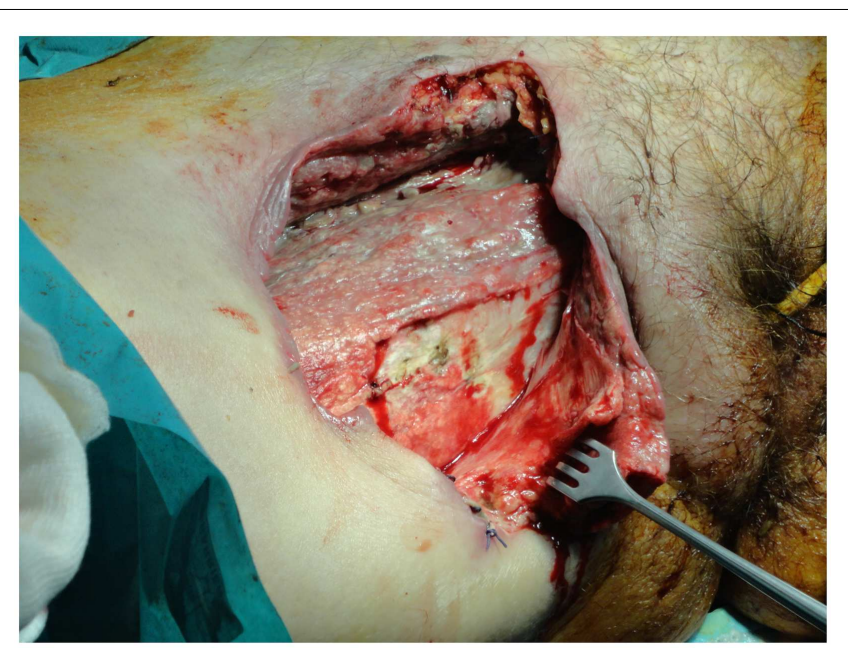

FIGURE 1 |The excision of the necrotic tissues should extend until healthy tissue is found, but should be limited to the edges of the infection.

ventricle incisions are made, keeping the wound open in order to allow drainage and to remove additional necrotic tissue.

Patients with NF should be closely monitored during the next $24 \mathrm{~h}$; surgical wounds and tissue viability should be checked. Complicated surgical wounds command a "second-look operation" with radical surgical debridement. Patients with NF can require from 5 up to 40 additional operations, depending on the timing of the first surgical debridement, the adequacy of the primary debridement and necrosectomy, signs of hemodynamic instability, and concomitant illnesses, all of which are associated with a high mortality rate (67). Evidence of hemodynamic instability demands immediate resuscitation, transfer to an intensive care unit, nutritional support, and enteral feeding.

The extent of tissue extracted depends on the body region, which is infected. As a general rule, debridement will extend until healthy tissue is found, though some authors recommend that excision should be limited to the edges of infection (68). The general consensus is that careful trimming of the potentially salvageable soft tissue is also required (while non-infected skin remains unattached) (Figure 1) (69).

Nutritional support is required from the first day of the patient's admission to hospital (preferably the ICU), to replace lost proteins and fluid from large wounds and/or the resultant toxic shock. Metabolic demands are similar to those of other major trauma or burns, which means that the patient needs twice the basic caloric requirements.

Necrotizing fasciitis of the abdominal wall requires special consideration. Skin incision must be performed in the longitudinal direction along the muscle-fascial layers of the inner abdominal wall until healthy fascia is found. Parallel or ventricle incisions are not performed because the bridges of skin and skin islands will not usually survive. Postoperative management of abdominal wall wounds involves serial dressing changes over the following days, until the wound is free of recurrent or ongoing infection. The use of a vacuum-assisted wound closing device (VAC) can also be helpful. Aggressive surgical debridement should be repeated in cases of infection progression across the deep fascial planes of the abdominal wall. The extension of infection into the bowel, resulting in bowel ischemia, bowel obstruction, and peritonitis, is not an uncommon phenomenon. In such cases, an exploratory laparotomy is needed to estimate the extent of infection inside the abdominal wall. A radical surgical debridement at the site of infection and the retroperitoneal site is performed, followed by partial bowel excision, depending on the part of the bowel (usually right colon), which has been infected. A diverting colostomy is performed with multiple drainages of the infected intra-abdominal fluid collections. Surgical management of colonic perforation complicated with peritonitis is a topic with considerable debate in the literature. Hartmann's resection has been considered the procedure of choice in cases with diffuse peritonitis and remains a safe technique for colectomy in a perforated colon, especially in elderly patients with multiple co-morbidities. As concerns NF, Hartmann's resection is particularly preferred, since it allows time for reconstruction of the abdominal wall defects, and the diverting colostomy can be closed at a second stage. The primary defect on the abdominal wall is usually large and is repaired with advanced flaps using an abdominoplasty technique, biological mesh, or skin grafts (70).

Fournier's gangrene also requires special consideration. A pressure sore, perineal abscess, or paraplegia frequently predispose to the spread of infection into the scrotum, inguinal region, and lower abdominal wall (Figure 2). An orchiectomy, cystostomy, or diverting colostomy is often required dependent on whether the infection has extended to the scrotum, perineal area, or lower abdominal wall, respectively. Surgical management includes wide tissue incision, radical debridement, and drainage of the areas involved (71). The wound is washed with hydrogen peroxide, saline, and $1 \%$ povidone iodine solution. Finally, it is covered with occlusive and adsorptive dressing with antiseptic properties. Again the use of VAC can accelerate the recovery period, providing clean surgical wounds. Once the patient is clinically and hemodynamically stable, they can be submitted to reconstructive surgery.

Necrotizing fasciitis of the extremities needs special consideration. The extent of debridement is very important as additional fasciotomies are needed in cases with compromised tissue viability (67). The amount of tissue that needs to be excised is a controversial issue, because the skin in the extremities usually appears normal.

A study by Andreasen et al. showed that despite a normal external appearance soft tissue in patients with NF has extensive vascular microthromboses as well as vasculitis. The risk of full-thickness necrosis is high, and this can complicate a primary treated surgical wound (72). Consequently, it is recommended that clinicians also remove healthy soft tissue, bearing in mind that extremities showing NF may require amputation. The criteria for amputation have been recommended by Tang et al. (73), the most significant of which is extensive soft tissue necrosis with involvement of the underlying muscles and rapidly progressing infection with a large area of tissue necrosis. Other conditions that may justify amputation, are the presence of concurrent medical disease with high anesthetic risk (ASA score III and above), and the presence of shock 


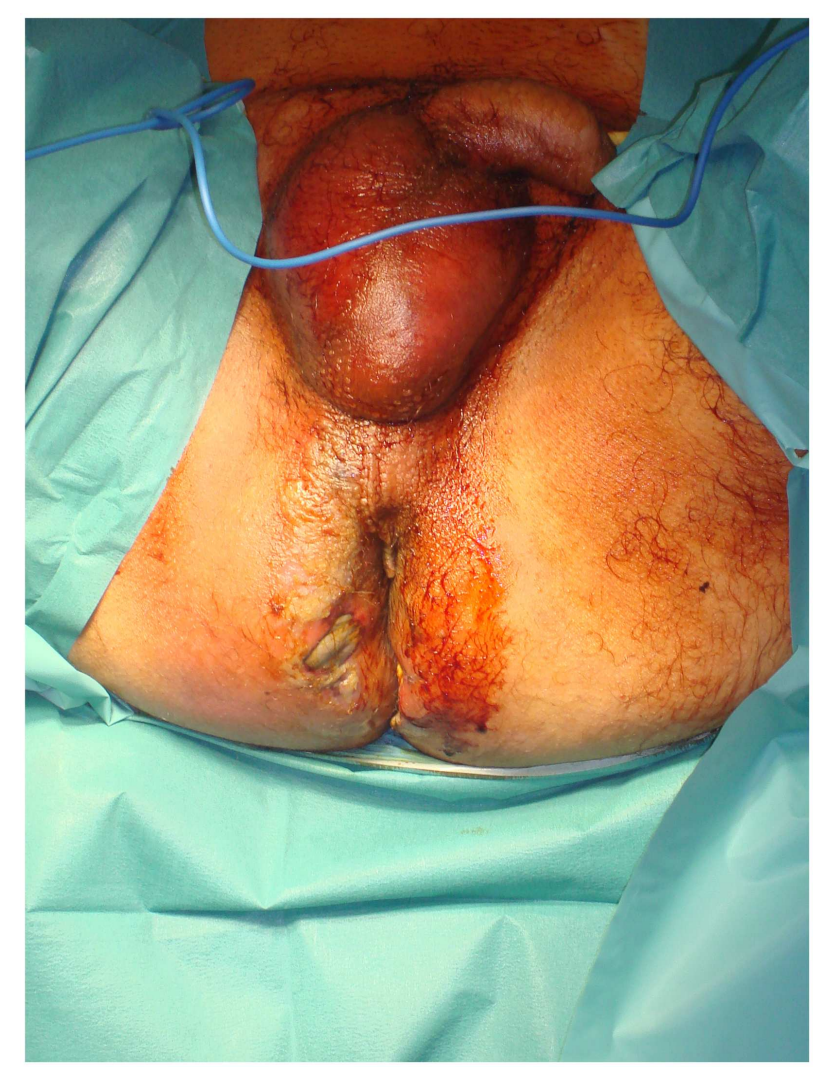

FIGURE 2 | A severe case of Fournier's gangrene with excessive erythema and edema in the perineal and gluteal regions as well as skin necrosis with bullae.

(toxic or cardiogenic) requiring treatment with more than one inotrope. Furthermore, concurrent vascular insufficiency further increases the need for amputation, especially when the patient is diabetic. Amputation is usually considered as a shorter procedure associated with less blood loss than a radical debridement. This explains why patients with hypotension and shock are best treated with amputation, as they cannot endure additional protracted operations. Studies have proved that, although amputation is not seen to reduce mortality, patients undergoing this procedure required fewer repeat operations, which is extremely important for patients presenting severe co-morbidities or a fulminant form of NF (74).

Recent reports of NF of the axilla require special consideration. A delay in surgical debridement can prove lethal, even more so than an infection in a common site (75). Tissue in the axillary region that is rich in blood vessels and lymphatics enables the infection to spread rapidly to distant sites. The clinician should keep in mind the need to avoid axillary contractures, after covering the exposed neurovascular network (75). Again, surgical reconstruction of the wound is essential for successful wound closure and should be planned after clinical stabilization $(76,77)$.

\section{USE OF VACUUM-ASSISTED CLOSURE DEVICE}

Lately, many surgeons worldwide have started using vacuumassisted closure (VAC) therapy for fast and effective wound closure

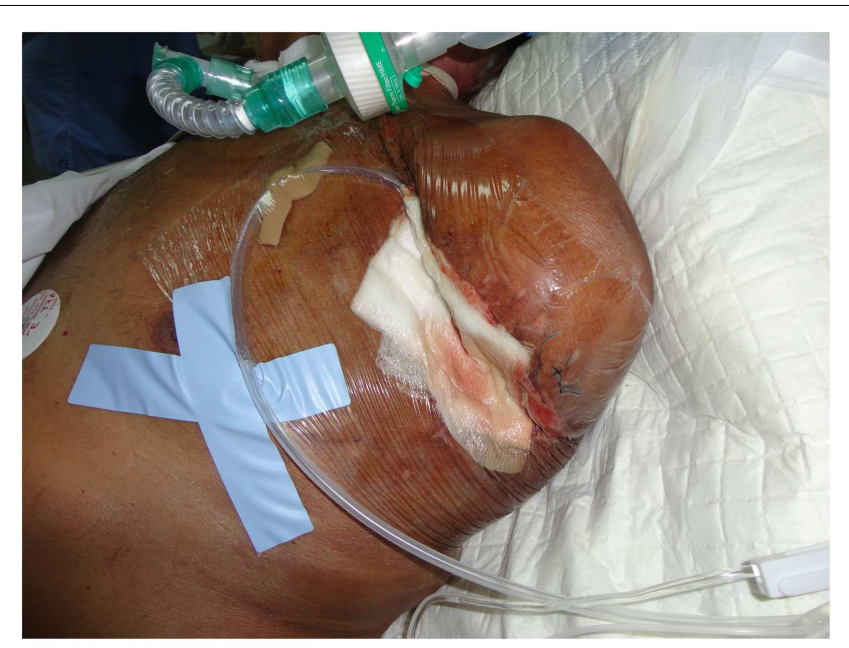

FIGURE 3 | After surgical debridement, the use of the VAC system helps wound healing by absorbing excess exudates; reducing localized edema, and finally drawing wound edges together.

(78). Several studies in the general surgery, orthopedic, and gynecological literature support the use of VAC devices. A VAC device consists of a sterile, open-cell foam sponge that is placed in the wound, the size of which is adjusted to the wound size. This is covered with a transparent adhesive drape to create an airtight environment. The sponge is connected to a portable vacuum pump by means of non-collapsible tubing. Evacuation is applied to the sponge using the pump, which provides continuous negative pressure. The VAC device improves wound healing by providing microstrain (Figure 3). Several randomized studies have demonstrated improved wound healing and a significant reduction of wound surface area in full-thickness wounds treated with VAC devices as compared to conventional gauze therapy (79).

The VAC dressing must be changed every $24-72 \mathrm{~h}$. VAC therapy has several benefits in wound management, with wound area reduction and formation of granulation tissue being the most prominent. Other benefits, such as effective wound cleaning and the ability to remove the exudate render VAC a promising adjuvant therapy for wound closure.

\section{FUTURE THERAPIES}

As a life-threatening condition, NF demands new management strategies. Unfortunately, there are no single new therapies that can manage NF; they all seem to play an assistive role. Undoubtedly, the use of VAC has many benefits in wound healing, and it will be adopted by more physicians in the future.

A study by Anaya et al. (13) highlighted the role of IVIG in the treatment of NSTI, especially if NSTI is associated with group A streptococcal infection. The authors concluded that the use of IVIG seemed rational in patients with group A streptococcal infection who developed streptococcal toxic shock syndrome and in those with a high mortality risk (advanced age, hypotension, and bacteremia). However, relevant studies investigating its use are contentious and difficult to compare because of the small number of patients and the different methodologies used. 
An interesting study by $\mathrm{Lu}$ et al. (80) showed that kallistatin, originally found to be a tissue kallikrein-binding protein, can increase the survival of group A streptococcus infected mice. The researchers concluded that kallistatin significantly increased the survival rate of GAS-infected mice, and also reduced local skin damage and bacterial counts. Moreover, its use improved infiltrating cell viability in the local infection site, as well as bacterial clearance activity of immune cells (81). The efficiency of intracellular bacterial killing in neutrophils was directly enhanced by kallistatin administration. Several inflammatory cytokines, including tumor necrosis factor alpha, interleukin $1 \beta$, and interleukin 6 , in local infection sites were reduced by kallistatin. Furthermore, kallistatin treatment was reported to reduce vessel leakage, bacteremia, and liver pathology after local infection. However, further studies are warranted before safe conclusions can be drawn concerning its use in gas-forming infections, such as NF (82).

\section{CONCLUSION}

Necrotizing fasciitis is a rare but life-threatening condition, with a high mortality rate (median mortality $32.2 \%$ ) that approaches $100 \%$ without treatment. Numerous conditions are associated with this pathology, such as diabetes mellitus, immunosuppression, chronic alcohol disease, chronic renal failure, and liver cirrhosis, which can be conductive to the rapid spread of necrosis, and increase in the mortality rate. The diagnosis of NF is difficult and the differential diagnosis between NF and other necrotizing soft tissue infections more so. However, the clinician should do their utmost to secure the diagnosis of NF, as a delay in diagnosis can be fatal, and septic shock is inevitable if the disease remains untreated. The characteristic of NF is the clinical status change over time. The early clinical picture includes erythema, swelling, tenderness to palpation, and local warmth; once the infection develops, the infection site presents skin ischemia with blisters and bullae. The diagnosis of NF can be secured faster with the use of laboratorybased scoring systems, such as the LRINEC score or the FGSI score, especially in cases of Fournier's gangrene. However, the diagnosis is definitely established by performing explorative surgery at the infected site.

Management of the infection begins with antibiotic treatment. In the majority of cases with NF (70-90\%) the reasonable pathogens are two or more, suggesting the use of broad-spectrum antibiotics. The value of antibiotic treatment in NF is relatively low, and early and aggressive drainage and debridement is required. In NF of the extremities, the clinician should consider amputating the infected limb, although this will not reduce the risk of mortality. Finally, postoperative management of the surgical wound is important, along with proper nutrition of the patient. The use of VAC therapy in wound management has greatly improved the results of postoperative management.

\section{REFERENCES}

1. Levine EG, Manders SM. Life-threatening necrotizing fasciitis. Clin Dermatol (2005) 23:144-7. doi:10.1016/j.clindermatol.2004.06.014

2. Urschel JD, Takita H, Antkowiak JG. Necrotizing soft tissue infections of the chest wall. Ann Thorac Surg (1997) 64:276-9. doi:10.1016/S0003-4975(97) 00514-6

3. Kaafarani HM, King DR. Necrotizing skin and soft tissue infections. Surg Clin North Am (2014) 94:155-63. doi:10.1016/j.suc.2013.10.011
4. Descamps V, Aitken J, Lee MG. Hippocrates on necrotising fasciitis. Lancet (1994) 344:556. doi:10.1016/S0140-6736(94)91956-9

5. Pouteau C. Mémoire ou recherches sur les symptômes de la gangrene humide des Hôpitaux. In: Poueau C, editor. uvres posthumes. (Vol. 3), Paris: Ph-D Pierres (1783). p. 239-68.

6. Jones J. Surgical Memoirs of the War of the Rebellion: Investigation Upon the Nature, Causes and Treatment of Hospital Gangrene as Prevailed in the Confederate Armies 1861-1865. New York, NY: US Sanitary Commission (1871).

7. Fournier JA. Gangrene foudroyante de la verge. Med Pract (1883) 4:589-97.

8. Brewer GE, Meleney FL. Progressive gangrenous infection of the skin and subcutaneous tissues, following operation for acute perforative appendicitis: a study in symbiosis. Ann Surg (1926) 84:438-50. doi:10.1097/00000658192684030-00017

9. Wilson B. Necrotizing fasciitis. Am Surg (1952) 18:416-31.

10. Stevens DL, Tanner MH, Winship J. Severe group A streptococcal infections associated with a toxic shock-like syndrome and scarlet fever toxin A. N Engl J Med (1989) 321:1-7. doi:10.1056/NEJM198907063210101

11. Kaul R, McGeer A, Low DE, Green K, Schwartz B. Population based surveillance for group A streptococcal necrotizing fasciitis: clinical features, prognostic indicators, and microbiologic analysis of seventy-seven cases. Ontario Group A Streptococcal Study. Am J Med (1997) 103:18-24. doi:10.1016/S0002-9343(97) 00160-5

12. Goh T, Goh LG, Ang CH, Wong $\mathrm{CH}$. Early diagnosis of necrotizing fasciitis. $\mathrm{Br}$ J Surg (2014) 101:119-25. doi:10.1002/bjs.9371

13. Anaya DA, Dellinger EP. Necrotizing soft-tissue infection: diagnosis and management. Clin Infect Dis (2007) 44:705-10. doi:10.1086/511638

14. Schecter W, Meyer A, Schecter G. Necrotising fasciitis of the upper extremity. J Hand Surg (1982) 7:15-9. doi:10.1016/S0363-5023(82)80006-3

15. Eke N. Fournier's gangrene: a review of 1726 cases. Br J Surg (2000) 87:718-28. doi:10.1046/j.1365-2168.2000.01497.x

16. Anaya DA, McMahon K, Nathens AB. Predictors of mortality and limb loss in necrotizing soft tissue infections. Arch Surg (2005) 140:151-7. doi:10.1001/ archsurg.140.2.151

17. Espandar R, Sibdari SY, Rafiee E, Yazdanian S. Necrotizing fasciitis of the extremities a prospective study. Strategies Trauma Limb Reconstr (2011) 6:121-5. doi:10.1007/s11751-011-0116-1

18. Park K, Jung S, Jung Y, Shin J, Hwang J. Marine bacteria as a leading cause of necrotising fasciitis in coastal areas of South Korea. Am J Trop Med Hyg (2009) 80:646-50.

19. Roje Z, Roje Z, Matic D, Librenjak D, Dokuzovic S, Varvodic J. Necrotizing fasciitis: literature review of contemporary strategies for diagnosing and management with three case reports: torso, abdominal wall, upper and lower limbs. Word J Emerg Surg (2011) 23(6):46. doi:10.1186/1749-7922-6-46

20. Martinschek A, Evers B, Lampl L, Gerngroß H, Schmidt R, Sparwasser C. Prognostic aspects, survival rate, and predisposing risk factors in patients with Fournier's gangrene and necrotizing soft tissue infections: evaluation of clinical outcome of 55 patients. Urol Int (2012) 89:173-9. doi:10.1159/000339161

21. Yeung YK, Ho ST, Yen CH, Ho PC, Tse WL, Lau YK, et al. Wong factors affecting mortality in Hong Kong patients with upper limb necrotising fasciitis. Hong Kong Med J (2011) 17:96-104.

22. Clayton MD, Fowler JE Jr, Sharifi R, Pearl RK. Causes, presentation and survival of fifty-seven patients with necrotizing fasciitis of the male genitalia. Surg Gynecol Obstet (1990) 170:49-55.

23. Green JR, Dafoe DC, Raffin TA. Necrotizing fasciitis. Chest (1996) 110:219-28. doi:10.1378/chest.110.1.219

24. Kalaivani V, Hiremath BV, Indumathi VA. Necrotising soft tissue infection-risk factors for mortality. JClin Diagn Res (2013) 7:1662-5. doi:10.7860/JCDR/2013/ 5535.3240

25. Elliott DC, Kufera JA, Myers RA. NSTI-risk factors for mortality and strategies for management. Ann Surg (1996) 224:672-83. doi:10.1097/00000658199611000-00011

26. Sorensen MD, Krieger JN, Rivara FP, Klein MB, Wessells H. Fournier's gangrene: management and mortality predictors in a population based study. J Urol (2009) 182:2742-7. doi:10.1016/j.juro.2009.08.050

27. Rea WJ, Wyryck WJ Jr. Necrotizing fasciitis. Ann Surg (1970) 172:957. doi:10. 1097/00000658-197012000-00005

28. Morua AG, Lopez JA, Garcia JD, Montelongo RM, Guerra LS. Fournier's gangrene: our experience in 5 years, bibliographic review and assessment of the Fournier's gangrene severity index. Arch Esp Urol (2009) 62:532-40. 
29. Czymek R, Frank P, Limmer S, Schmidt A, Jungbluth T, Roblick U, et al. Fournier's gangrene: is the female gender a risk factor? Langenbecks Arch Surg (2010) 395:173-80. doi:10.1007/s00423-008-0461-9

30. Benjelloun el B, Souiki T, Yakla N, Ousadden A, Mazaz K, Louchi A, et al. Fournier's gangrene: our experience with 50 patients and analysis of factors affecting mortality. World J Emerg Surg (2013) 8:13. doi:10.1186/17497922-8-13

31. Angoules AG, Kontakis G, Drakoulakis E, Vrentzos G, Granick MS, Giannoudis PV. Necrotising fasciitis of upper and lower limb: a systematic review. Injury (2007) 38(Suppl 5):19-26.

32. Sudarsky LA, Laschinger JC, Coppa GF, Spencer FC. Improved results from a standardized approach in treating patients with necrotizing fasciitis. Ann Surg (1987) 206:661-5. doi:10.1097/00000658-198711000-00018

33. Ruiz-Tovar J, Córdoba L, Devesa JM. Prognostic factors in Fournier gangrene. Asian J Surg (2012) 35:37-41. doi:10.1016/j.asjsur.2012.04.006

34. Huang KF, Hung MH, Lin YS, Lu CL, Liu C, Chen CC, et al. Independent predictors of mortality for necrotizing fasciitis: a retrospective analysis in a single institution. J Trauma (2011) 71:467-73. doi:10.1097/TA.0b013e318220d7fa

35. Morgan MS. Diagnosis and management of necrotising fasciitis: a multiparametric approach. J Hosp Infect (2010) 75:249-57. doi:10.1016/j.jhin.2010.01.028

36. Weiss KA, Laverdière M. Group A Streptococcus invasive infections: a review. Can J Surg (1997) 40:18-25.

37. Souyri C, Olivier P, Grolleau S, Lapeyre-Mestre M. French network of pharmacovigilance centres. Severe necrotizing soft-tissue infections and nonsteroidal anti-inflammatory drugs. Clin Exp Dermatol (2008) 33:249-55. doi:10.1111/j. 1365-2230.2007.02652.x

38. Kimura AC, Higa JI, Levin RM, Simpson G, Vargas Y, Vugia DJ. Outbreak of necrotizing fasciitis due to Clostridium sordellii among black-tar heroin users. Clin Infect Dis (2004) 38:87-91. doi:10.1086/383471

39. Gauzit R. Necrotizing skin and soft tissue infections: definitions, clinical and microbiological features. Ann Fr Anesth Reanim (2006) 25:967-70. doi:10.1016/ j.annfar.2006.03.018

40. Bakleh M, Wold LE, Mandrekar JN, Harmsen WS, Dimashkieh HH, Baddour LM. Correlation of histopathologic findings with clinical outcome in necrotizing fasciitis. Clin Infect Dis (2005) 40:410-4. doi:10.1086/427286

41. Shimizu T, Tokuda Y. Necrotizing fasciitis. Intern Med (2010) 49:1051-7. doi:10.2169/internalmedicine.49.2964

42. Frazee BW, Fee C, Lynn J, Wang R, Bostrom A, Hargis C, et al. Communityacquired necrotizing soft tissue infections: a review of 122 cases presenting to a single emergency department over 12 years. J Emerg Med (2008) 34:139-46. doi:10.1016/j.jemermed.2007.03.041

43. DiNubile MJ, Lipsky BA. Complicated infections of skin and skin structures: when the infection is more than skin deep. J Antimicrob Chemother (2004) 53(Suppl 2):37-50.

44. Sultan HY, Boyle AA, Sheppard N. Necrotizing fasciitis. BMJ (2012) 345:4274. doi:10.1136/bmj.e4274

45. Wang YS, Wong CH, Tay YK. Staging of necrotizing fasciitis based on the evolving cutaneous features. Int J Dermatol (2007) 46:1036-41. doi:10.1111/j.13654632.2007.03201.x

46. Dworkin M, Westercamp M, Park L, McIntyre A. The epidemiology of necrotising fasciitis including factors associated with death and amputation. Epidemiol Infect (2009) 137:1609-14. doi:10.1017/S0950268809002532

47. Majeski J, Majeski E. Necrotising fasciitis: improved survival with early recognition by tissue biopsy and aggressive surgical treatment. South Med J (1997) 90:1065-8. doi:10.1097/00007611-199711000-00001

48. Wong C, Khin L. Clinical relevance of the LRINEC (laboratory risk indicator for necrotizing fasciitis) score for assessment of early necrotizing fasciitis. Crit Care Med (2005) 33:1677. doi:10.1097/01.CCM.0000170199.43624.B8

49. Majeski JA, Alexander JW. Early diagnosis, nutrition support, and immediate extensive debridement improve survival necrotizing fasciitis. Am J Surg (1983) 145:785-7.

50. Mizokami F, Furuta K, Isogai Z. Necrotizing soft tissue infections developing from pressure ulcers. J Tissue Viability (2014) 23:1-6. doi:10.1016/j. jtv.2013.11.001

51. Wong CH, Khin LW, Heng KS, Tan KC, Low CO. The LRINEC (laboratory risk indicator for necrotizing fasciitis) score: a tool for distinguishing necrotizing fasciitis from other soft tissue infections. Crit Care Med (2004) 32:1535-41. doi:10.1097/01.CCM.0000129486.35458.7D
52. Chao WN, Tsai SJ, Tsai CF, Su CH, Chan KS, Lee YT, et al. The laboratory risk indicator for necrotizing fasciitis score for discernment of necrotizing fasciitis originated from Vibrio vulnificus infections. J Trauma Acute Care Surg (2012) 73:1576-82. doi:10.1097/TA.0b013e318270d761

53. Thomas AJ, Meyer TK. Retrospective evaluation of laboratory-based diagnostic tools for cervical necrotizing fasciitis. Laryngoscope (2012) 122:2683-7. doi:10.1002/lary.23680

54. Wong C, Wang Y. The diagnosis of necrotizing fasciitis. Curr Opin Infect Dis (2005) 18:101-6. doi:10.1097/01.qco.0000160896.74492.ea

55. Monneuse O, Gruner L, Barth X, Malick P, Timsit M, Gignoux B, et al. Gas gangrene of the abdominal wall due to underlying GI pathology: seven cases. J Chir (Paris) (2007) 144:307-12. doi:10.1016/S0021-7697(07)91959-6

56. Wang T, Hung CR. Role of tissue oxygen saturation monitoring in diagnosing necrotizing fasciitis of the lower limbs. Ann Emerg Med (2004) 44:222-8. doi:10.1016/j.annemergmed.2004.03.022

57. Nagano N, Isomine S, Kato H, Sasaki Y, Takahashi M, Sakaida K, et al. Human fulminant gas gangrene caused by Clostridium chauvoei. J Clin Microbiol (2008) 46:1545-7. doi:10.1128/JCM.01895-07

58. Marron CD. Superficial sepsis, cutaneous abscess and necrotizing fasciitis. In: Brooks A, Mahoney PF, Cotton BA, Tai N, editors. Emergency Surgery. Oxford, UK: Blackwell Publishing (2010). p. 115-23.

59. Lille ST, Sato TT, Engrav LH, Foy H, Jurkovich GJ. Necrotizing soft tissue infections: obstacles in diagnosis. J Am Coll Surg (1996) 182:7-11.

60. Zimbelman J, Palmer A, Todd J. Improved outcome of clindamycin compared with beta-lactam antibiotic treatment for invasive Streptococcus pyogenes infection. Pediatr Infect Dis J (1999) 18:1096. doi:10.1097/00006454199912000-00014

61. Mulla ZD. Treatment options in the management of necrotising fasciitis caused by group A Streptococcus. Expert Opin Pharmacother (2004) 5:1695-700. doi:10.1517/14656566.5.8.1695

62. Naqvi GA, Malik SA, Jan W. Necrotizing fasciitis of the lower extremity: a case report and current concept of diagnoses and management. Scan J Trauma Resusc Emerg Med (2009) 17:28. doi:10.1186/1757-7241-17-28

63. Norrby-Teglund A, Muller MP, McGeer A. Successful management of severe group A streptococcal soft tissue infection using an aggressive medical regimen including intravenous polyspecific immunoglobulin together with a concervative surgical approach. Scand J Infect Dis (2005) 37:166.

64. Kaul R, McGeer A, Norrby-Teglund A. Intravenous immunoglobulin therapy for streptococcal toxic shock syndrome; a comparative observational study. Clin Infect Dis (1998) 28:800-7. doi:10.1086/515199

65. Mok MY, Wong SY, Chan TM. Necrotizing fasciitis in rheumatic diseases. Lupus (2006) 15:380-3. doi:10.1191/0961203306lu2314cr

66. Wong KC, Shih CH. Necrotizing fasciitis of the extremities. J Trauma (1992) 32:179-82. doi:10.1097/00005373-199202000-00011

67. Roje Z, Roje Ž, Eterović D, Družijanić N, Petričević A, Roje T, et al. Influence of adjuvant hyperbaric oxygen therapy on short-term complications during surgical reconstruction of upper and lower extremity war injury: a retrospective cohort study. Croat Med J (2008) 49:224-32. doi:10.3325/cmj.2008.2.224

68. Sarani B, Strong M, Pascual J, Schwab CW. Necrotizing fasciitis: current concept and review of the literature. J Am Coll Surg (2009) 208:279-88. doi:10.1016/j.jamcollsurg.2008.10.032

69. Wong CH, Yam AK, Tan AB, Song C. Approach to debridement in necrotizing fasciitis. Am J Surg (2008) 196:19-24. doi:10.1016/j.amjsurg.2007.08.076

70. Brafa A, Grimaldi L, Brandi C, Nisi G, Calabro M, Campa SA, et al. Abdominoplasty as a reconstructive surgical treatment of necrotizing fasciitis of the abdominal wall. J Plast Reconstr Aesthet Surg (2009) 62:136-9. doi:10.1016/j.bjps.2008. 07.031

71. Azize KIC, Ylmaz Ali KL. Fournier's gangrene: etiology, treatment, and complications. Ann Plast Surg (2001) 147:523-7.

72. Andreasen TJ, Green SD, Childers BJ. Massive infectious soft tissue injury: diagnosis and management of necrotizing fasciitis and purpura fulminans. Plast Reconstr Surg (2001) 107:1025-34. doi:10.1097/00006534-20010401000019

73. Tang WM, Ho PL, Fung KK, Yuen KY, Leong JC. Necrotising fasciitis of a limb. J Bone Joint Surg Br (2001) 83:709-14. doi:10.1302/0301-620X.83B5.10987

74. Wong CH, Chang HC, Pasupathy S, Khin LW, Tan JL, Low CO. Necrotizing fasciitis: clinical presentation, microbiology, and determinants of mortality. J Bone Joint Surg Am (2003) 85:1454-60. 
75. Adachi K, Tsutsumi R, Yoshida Y, Watanabe T, Nakayama B, Yamamoto O. Necrotizing fasciitis of the breast and axillary regions. Eur J Dermatol (2012) 22:817-8.

76. Yamasaki O, Nagao Y, Sugiyama N, Otsuka M, Iwatsuki K. Surgical management of axillary necrotizing fasciitis: a case report. J Dermatol (2012) 39:309-11. doi:10.1111/j.1346-8138.2011.01456.x

77. Netscher DT, Baumholtz MA, Bullocks J. Chest reconstruction: II. Regional reconstruction of chest wall wounds that do not affect respiratory function (axilla, posterolateral chest, and posterior trunk). Plast Reconstr Surg (2009) 124:427-35. doi:10.1097/PRS.0b013e3181bf8323

78. Silberstein J, Grabowski J, Parsons JK. Use of a vacuum-assisted device for Fournier's gangrene: a new paradigm. Rev Urol (2008) 10:76-80.

79. Moues CM, van den Bemd GJ, Heule F, Hovius SE. Comparing conventional gauze therapy to vacuum-assisted closure wound therapy: a prospective randomised trial. J Plast Reconstr Aesthet Surg (2007) 60:672-81. doi:10.1016/j. bjps.2006.01.041

80. Lu SL, Tsai CY, Luo YH, Kuo CF, Lin WC, Chang YT, et al. Kallistatin modulates immune cells and confers anti-inflammatory response to protect mice from group A streptococcal infection. Antimicrob Agents Chemother (2013) 57:5366-72. doi:10.1128/AAC.00322-13

81. Nisbet M, Ansell G, Lang S, Taylor S, Dzendrowskyj P, Holland D. Necrotising fasciitis: review of 82 cases in South Auckland. Intern Med J (2011) 47:543-8. doi:10.1111/j.1445-5994.2009.02137.x
82. Dalsgaard A, Frimodt-Moller N, Bruun B, Hoi L, Larsen JL. Clinical manifestations and molecular epidemiology of Vibrio vulnificus infections in Denmark. Eur J Clin Microbiol Infect Dis (1996) 15:227-32. doi:10.1007/BF01591359

Conflict of Interest Statement: The authors declare that the research was conducted in the absence of any commercial or financial relationships that could be construed as a potential conflict of interest.

Received: 08 April 2014; paper pending published: 31 May 2014; accepted: 24 August 2014; published online: 29 September 2014.

Citation: Misiakos EP, Bagias G, Patapis P, Sotiropoulos D, Kanavidis Pand Machairas A (2014) Current concepts in the management of necrotizing fasciitis. Front. Surg. 1:36. doi: $10.3389 /$ fsurg.2014.00036

This article was submitted to Visceral Surgery, a section of the journal Frontiers in Surgery.

Copyright (c) 2014 Misiakos, Bagias, Patapis, Sotiropoulos, Kanavidis and Machairas. This is an open-access article distributed under the terms of the Creative Commons Attribution License (CC BY). The use, distribution or reproduction in other forums is permitted, provided the original author(s) or licensor are credited and that the original publication in this journal is cited, in accordance with accepted academic practice. No use, distribution or reproduction is permitted which does not comply with these terms. 